\title{
An Example of Variations in Oceanic Circulation in Response to the Variations in Wind Field ${ }^{*}$
}

\author{
By
}

\author{
Kozo YoshIDA**
}

\begin{abstract}
An example of seasonal variations in surface oceanic circulation is revealed from the data off the California coasts. An account for them may probably be given by a simple model in which the rearrangement of density through wind-induced vertical motion is essential to the changes. This mechanism might also apply more widely to annual variations in oceanic circulation in response to those in atmospheric pressure field. An emphasis may be placed on the point that the variations in currents are in quasi-stationary balance with pressure field in the ocean surface layer which varies as integral effect of winds. A possibility of quantitative prediction might be suggested.
\end{abstract}

\section{Introduction}

It should be a matter of great difficulty at present to attempt to deal with variations in oceanic circulation. This involves time constants of the ocean circulation which so far have not been well known. A lack of this knowledge is primarily ascribed to the difficulties in doing observations with sufficient density and simultaneity at sea. It is therefore required at first that more observations should be made in this direction as they are being activated in recent years. Nevertheless, it might be desirable to get some rough ideas of such variations, even ahead of observations, considering that any individual data which are collected to be analyzed must represent various particular phases of ever-changing pattern of the phenomena. In this connection, the author is inclined to feel that some of the foregoing theoretical approaches might be misleading(1) and might require

* Received Aug. 3, 1935.

** Geophysical Institute, Tokyo University, Tokyo.

(1) It seems somewhat misleading to apply the following simplified models to the variations of periods longer than several days:

(1) The acceleration terms are formally included in the dynamical equations, whereas the changes in mass distributions are essentially disregarded or not critically taken into consideration;

(2) Quasi-static equilbrium is assumed to apply to the variations in circulation patterns as well as in mass distributions. discussions.

For an annual variation, for instance, the dynamical balance would approximately be quasi-stationary without acceleration, except for the boundary zone such as close to coasts. Thus the instantaneous field of surface water cirulation which varies season to season would be well represented as a geostrophic flow, nearly in phase with the corresponding instantaneous surface slope. However, this does not mean that the currents respond to winds quasi-steadily, since readjustment of pressure field to winds will require some considerable time length. Mass distributions are changing during a large part of the period when vertical motion is induced by winds, and thus the surface current varies as an integral effect of winds. The time lag may therefore be mainly due to the time required for mass readjustment and not due to the acceleration.

In the waters off the California coasts, the observations taken by serial cruises at the Scripps Institution of Oceanography appear to have confirmed the facts that northerly California Current develops inshore during periods of upwelling. In the absense of prevailing winds that cause upwelling, a countercurrent (northward) would appear on the coastal side. These features of seasonal changes in surface currents are consistently revealed by the preceding observations. Some examples 

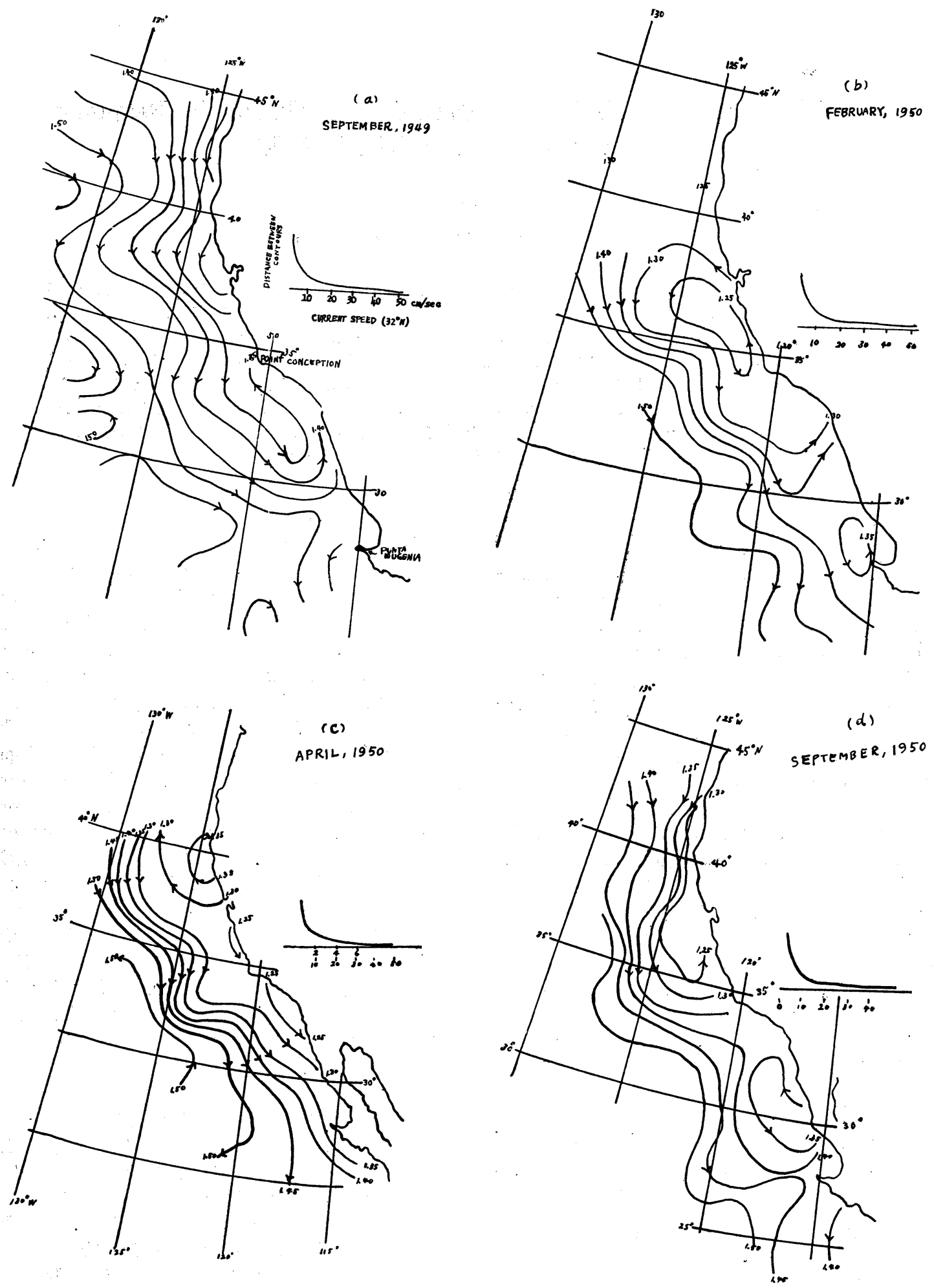

Fig. 1. Dynamic Height Anomalies (0 over 1000 decibars) : Contour interval is 0.05 dynamic meters, and the current direction is as indicated by arrows: (a) CCOFI Cruise 4909 (MLR, cruise 7) September 5 to September 18, 1949. (b) CCOFI Cruise 5002 (MLR, cruise 11) January 31 to February 19, 1950. (c) CCOFI Cruise 5004 (MLR, cruise 13) April 3 to 17, 1950. (d) CCOFI Cruise 5009 (MLR, cruise 18) September 6 to 22, 1950. 
are illustrated in Figs. 1a to 1d, taken from recent CCOFI (California Cooperative Oceanic Fisheries Investigation) cruise results. The cruises in 1937 and in 1938 also reveal remarkably consistent pictures. Refer to those figures* prepared by Sverdrup and Allen (1939) and those by Sverdrup and Fleming (1941). It has also been indicated that seasonal occurrence of upwelling seems to be in phase with seasonal changes in areas of positive wind stress curl off the coast. (Yoshida et al, 1955). The annual variations in mean sea level along the coasts have been found to be closely related to those in the high pressure field over the North Pacific (Jacobs, 1939). Recently, Munk, Pattullo, Revelle and Strong have confirmed that the annual variations in mean sea level are in satisfactory agreement with those in density field under an isostatic adjustment within an upper 400 meter layer.

These observed facts which have been accumulated in the California waters appear to support the idea outlined above at least in the cases of this current system, and might further enable us to go into somewhat quantitative discussions on variations in the currents. A possibility of forecasting oceanographic conditions might be suggested. Not only to the California current system, but also to the larger-scale circulation system, the similar mechanism might apply.

\section{A Model for California Current System}

For simplicity, let us take $x$ axis eastward, $y$ axis northward and $z$ axis vertically downward, and assume the coastline extends north to south. Except for the narrow zone close to the coast, some $50 \mathrm{~km}$ from coast, the dynamical equation for

* Refer to

(1) Fig. 4 (Cruise 1, March 25-April 3, 1937), Fig. 6 (Cruise 3, June 22-July 2, 1937), in the paper by Sverdrup and Fleming (1941), and

(2) Fig. 39 (Cruise 2, April, 1938),

Fig. 41 (Cruise 4, August, 1938),

Fig. 43 (Cruise 6, December, 1938),

in the paper by Sverdrup and Allen (1939). the upper mixed layer may be given by

$$
\begin{gathered}
-f v=-\partial p / \partial x \\
f u=-\partial p / \partial y+\tau y / H
\end{gathered}
$$

where $u$ and $v$ are eastward and northward component of velocity respectively, $f$ is Coriolis parameter, $p$ is pressure, $\tau_{y}$ is northward component of wind stress, $H$ is a mean depth of upper mixed layer.

From the above equations, vertical velocity $w_{h}$ at a thermocline $(z=h)$ is seen, to a first approximation, to be

$$
w_{h} \sim-(1 / f) \partial^{\top} y / \partial x
$$

provided that planetary vorticity $\beta v$, where $\beta=d f / d y$, is neglected, as could be justified for the surface current in the areas concerned (Yoshida et al, 1955). From the condition of nearly isostatic balance :**

$$
\partial p / \partial x \sim g \Delta \rho(\partial h / \partial x)
$$

where $\Delta \rho$ is a difference between the mean density in surface layer and in lower layer, and from the condition of mass conservation :

$$
u_{h} \sim \partial h / \partial t
$$

it follows that the change in velocity component $v$ is given by

$$
\partial v / \partial t \sim(g \Delta \rho / f)\left(\partial w_{h} / \partial x\right) \sim-\left(g \Delta \rho / f^{2}\right)\left(\partial^{2} \tau_{y} / \partial x^{2}\right)
$$

This eq. (6) implies that southward current will be accelerated during the period when upwelling takes place inshore. Combined this together with those features of seasonal variations in the wind field and in vertical motion off the California coasts which have been indicated by the author (Yoshida and Mao, 1955), it may be considered that the present model may represent observed features of the variations satisfactorily.

* From the examinations of cruise data off the California coast, February to May, 1949, and of the wind conditions over the areas off the coasts, Defant estimated that the sea surface would adjust itself to the wind within one or two days and that this quasistationary state would be maintained as long as the wind continues. (Albert Defant, Memorandum concerning the work of the Marine Life Research Program, La Jolla, 1950). 
As mentioned before, the seasonal changes in $w$ occur quasisteadily as winds vary season to season, and will result in the seasonal changes in thickness of mixed layer which associate the changes in surface currents. ${ }^{(2)}$ Now, quasi-static current field involves wind-induced vertical motion, which responds to winds without much delay, perhaps less than several days. The changes in surface currents are thus "caused" by non-uniformity in wind stress curl through the mechanism of vertical motion. The time lag for the response of the ocean surface circulation is due to the time required to establish a new mass field which itself is brought about by horizontal divergence in the basic currents. When winds vary season to season, for exmple, the surface currents vary with time lag of order of three months in absence of lateral diffusion. It appears, on the other hand, that the outstanding features in the variations revealed by observations off the California coasts may suggest the lag to be less, at most one to two months. The role of lateral diffusion may therefore be suggested, and (5) may be replaced, as previously derived (Yoshida, 1955) by

$$
w_{h} \sim \partial h / \partial t-B \partial^{2} h / \partial x^{2},
$$

where $B$ is a coefficient of lateral mixing. In fact, the second term indicating mixing will be quite comparable to the first when the annual variations are concerned. If we take $\sigma \sim 2 \times 10^{-7}$ (period: 1 year), $\mathrm{k} \sim 6 \times 10^{-8}$ (wavelength: $10^{3} \mathrm{~km}$ ) and $\mathrm{B} \sim 2 \times 10^{7}$, the phase lag will be

$$
\tan ^{-1} \frac{\sigma}{B k^{2}} \sim 70^{\circ} \sim 2.3 \text { months }
$$

where $\sigma$ and $k$ denote frequency and wave number of the disturbance, respectively. Although the matter may critically depend upon a magnitude of $B$ which is not well

(2) Time change in vorticity produced in this manner, however, would be still negligibly small compared with each of horizontal divergence and wind stress curl. Although the secondary variable currents will produce the corresponding additional changes in vertical velocity, this additional divergence is negligible in surface layer. known, it appears that the time lag for the wave of significant annual range will perhaps be one to two months. The corresponding range of annual variations in $v$ is found approximately to be $20 \mathrm{~cm} / \mathrm{sec}$, which may be in fair agreement with observed conditions encountered in the California waters. Typical examples of the features are clearly illustrated in Figs. 1a to 1d.

Within a narrow strip close to the coast, perhaps within 50 to $100 \mathrm{~km}$ from the coast, time changes in relative vorticity will be considerable (Yoshida, 1955), and lateral mixing will be essential to the variations probably due to large internal waves, horizontal eddies and large variability itself in the current, so that minor fluctuation in winds may result in relatively quick response of coastal currents which without much delay establish quasi-static equilibrium.

In lower layers of California waters, it appears to have been indicated that subsurface north-south transport changes from season to season nearly in phase with the corresponding changes in wind stress curl (Yoshida et al, 1955). The processes of adjustment might be represented by

$$
\partial^{2} v_{2} / \partial x \partial t+\beta v_{2}=\left(f / H_{2}\right) \operatorname{curl} \tau
$$

where $v_{2}$ is a meridional component of velocity in lower layer, and $H_{2}$ is a thickness of the lower layer.

Probably, in the current system where north-south component of velocity is predominant, the response of the current to winds is comparatively rapid, say, with a phase lag of order of a month or less. The response of the flow at depths greater than $500 \mathrm{~m}$ will, however, be much slower.

\section{Other Current Systems}

The Kuroshio, Gulf Stream, and perhaps whole oceanic circulation may vary in somewhat similar way, though more complicated conditions may be encountered in different areas. The detailed discussions in individual cases are not attempted in the present note, because reliable data, ocean- 
ographical as well as meteorological, are not readily available at hand. Only some general remarks may be made briefly. As seen from (6), upwelling on the left and sinking on the right of the stream looking in direction of flow will strengthen the main stream. Such conditions will be favored when the atmospheric high pressure is on the right, and the low on the left. The magnitude of canges in current speed is measured by $\frac{\partial^{2} \tau_{y}}{\partial x^{2}}$, that is, roughly by the relative intensity of the high and low, and also by the distance between them. Hence considerable fluctuations in currents may occur in the boundary regions between atmospheric high and low, where, in addition to the above effects, the direction of the winds roughly coincides with the current and a slight change in position or intensity of the atmospheric system might cause considerable variations in the oceanic currents. Now, it might be supposed that annual or yearly variations in the location and intensity of the large-scale atmospheric pressure system would cause the large-scale variations in the oceanic circulation over the wide areas of the oceans. For example, (3) nearly simultaneous changes in the oceanographic conditions on both sides of the North Pacific, off Japan and off California, might be caused by yearly changes in the position or intensity of the North Pacific High. These are as yet only speculative and the future works in cooperation between the western and the eastern portions of the Pacific would reveal the features.

In some relation to this, it might be worth mentioning to remark on the variations in the currents which have been reported in varions occasions of the passage of storms, or in asoociation with some atmospheric pressure field of shorter duration. It appears that the Gulf Stream and Kuroshio are intensified in the cases when the high pressure is offshore and the low is in coastal side of the Stream. It has also

(3) Similar idea has been presented by Paul $\mathrm{L}$. Horrer, the Scripps Institution of Oceanography, at the California Sardine Conference, July, 1953, Santa Maria, Calif. been observed that the northward current is intensified on the right hand side of the course of the storm. The variations associated with passage of storms or meteorological disturbances of duration of a week or shorter are somewhat different in their behaviors from those discussed in this paper. Mass redistributions may be done only by part before the atmospheric disturbance is removed. They should also be of practical importance on fishery problems and should require further studies by specifically planned observations.

In concluding the present discussions, it might be mentioned that some of the characteristic changes in California Current System could be predicted on the basis of oceanographic data, say, collected one month earlier.

\section{Conclusion and Discussions}

It should be too early to draw any conclusion at to seasonal fluctuations in the oceanic circulation on the basis of more or less hypothetical theory. What we need now indeed should, as every one does realize, be the more and better observations.

The outstanding features in seasonal changes in surface current system off the California coasts which have been revealed by the observations during years appear to suggest that the essential mechanism might be rather simple. The theory developed in the present paper seems to account for the main features of the variations, not only qualitatively but also quantitatively. For such system, dynamical equations and vorticity equations would merely represent the quasistationary equilibrium between the acting forces. Of course, they cannot alone tell how thecurrents change in response to wind fluctu ations. The changes could only be predicted by the prediction equation like (6) which is derived under certain assumptions concerning the changes in mass distributions in response to the changes in wind conditions. The results from the monthly cruises in the California waters appear to confirm that the assumptions may approximately be justified and that the prediction equation may be valid to a first approxi- 
mation. Its validity might fail in the other regions of the oceans, where different mechanism may prevail and data may not readily be available.

\section{Achnowledments}

The writer is indebted deeply to Paul. L. Horrer and Joseph L. Reid, who gave him, during his stay at La Jolla, many valuable suggestions and informations of the data in the California current regions.

\section{References}

Jacobs, W. C., 1939. Sea level departures on the California coast as related to the dynamics of the atmcsphere over the North Pacific Ocean. J. Mar. Res. 2 (3) : 181-194.

Munk, W. H., J. G. Pattullo, R. R. Revelle, and
Elizabeth Strong, 1954. The anual change in sea level. (In press)

Sverdrup, H. U., and W. E. Allen, 1939. Distribution of diatoms in relation to the character of water masses and currents off Southern California in 1938. J. Mar. Res. 2 (2) : 131-144.

Sverdrup, H. U., and R. H. Fleming, 1941. The waters off the coast of Southern California, March to July, 1937. Scripps Inst. Oceanogr., Bull. 4 (10) : 261-378.

Yoshida, Kozo, and Han-Lee Mao, 1955. A new measurement of upwelling with examples in the California current region. (In press)

Yoshida, Kozo, 1955a. Coastal upwelling off the California coast. (In press)

1955b. A discussion on responses of the oceanic circulation in response tc a fluctuating wind stress. (unpublished)

1955c. Interaction between the mixed layer and the lower layer. (uupublished) 